\title{
Diversite Floristique Et Structure Des Fragments Forestiers Du Sud-Ouest Du Parc National De La Comoe Apres Les Conflits Des Annees 2000 En Côte d'Ivoire
}

\author{
Yéo Zahana Seydou \\ Sangne Yao Charles \\ Barima Yao Sadaiou Sabas \\ Bamba Issouf \\ Kouakou Kouassi Apollinaire \\ Unité de Formation et de Recherche en Environnement, \\ Université Jean Lorougnon Guédé, Daloa, Côte d’Ivoire
}

doi: 10.19044/esj.2016.v13n2p421 URL:http://dx.doi.org/10.19044/esj.2016.v13n2p421

\begin{abstract}
Côte d'Ivoire, like many countries in Africa, has experienced a decade of crises that has not been without consequences for the natural formations in general and protected areas in particular. The objective of this study is to evaluate the floristic diversity and structure of forest fragments Southwest of National Park of Comoé after conflicts. The sampling methodology was to inventory all taxa along 500 meters long and a width of $10 \mathrm{~m}$ (5 m either side of the main line) installed in each forest fragment. In addition to the inventory of taxa, we measured the circumference of all trees at least $30 \mathrm{~cm}$ with their corresponding pitch. A total of 12 transects were sampled in 12 fragments of forest. Following these inventories, 256 plant species have been identified, the most abundant belong to the families of Rubiaceae and Fabaceae. 23 species have been recognized as having a special status, including Salacia miegei N. Halle and Uvaria tortilis A. Chev. Ex Hutch. \& Dalziel are endemic to the Côte d'Ivoire. Nine (9) others species have been identified as species of high commercial value for the quality of their wood. The forest formations are very dense with more than 550 trees / ha. It appears that the fragments contain few large trees. Despite the successive crises, it appears that forest fragments Southwest National Park Comoé still have good potential floristic.
\end{abstract}

Keywords: Comoé National Park, forest fragments, floristic diversity, special status species. 


\section{Résumé}

La Côte d'Ivoire, à l'instar de nombreux pays en Afrique, a connu une décennie de crises qui n'a pas été sans conséquences sur les formations naturelles en général et sur les aires protégées en particulier. L’objectif de cette étude est d'évaluer la diversité floristique, ainsi que la structure des fragments forestiers du Sud-Ouest du Parc National de la Comoé après les conflits. La méthode d'échantillonnage a consisté à inventorier tous les taxons sur des transects rectilignes de $500 \mathrm{~m}$ de longueur sur une largeur de $10 \mathrm{~m}$ (soit $5 \mathrm{~m}$ de part et d'autre de la ligne principale) posés dans chaque fragment forestier. Outre l'inventaire des taxons, la circonférence de tous les arbres d'au moins $30 \mathrm{~cm}$ avec leur hauteur correspondante ont été mesurées. Au total, 12 layons ont été échantillonnés dans 12 fragments forestiers. A l'issue de ces inventaires, 256 espèces végétales dont les plus abondantes appartiennent aux familles des Rubiaceae et Fabaceae, ont été recensées. 23 espèces ont été reconnues comme ayant un statut particulier, parmi lesquelles Salacia miegei N. Halle et Uvaria tortilis A. Chev. Ex Hutch. \& Dalziel sont endémiques à la Côte d'Ivoire. 9 autres espèces ont été reconnues comme espèces de grande valeur commerciale pour la qualité de leur bois. Les formations forestières sont très denses avec plus de 550 arbres/ha. Il ressort que les fragments contiennent peu d'arbres de grandes tailles. Malgré les crises successives, les fragments forestiers du Sud-Ouest du Parc National de la Comoé présentent encore un bon potentiel floristique.

Mots clés: Parc national de Comoé, fragments forestiers, diversité floristique, espèces à statut particulier.

\section{Introduction}

Les aires protégées possèdent des racines historiques lointaines. Elles ont existé sous plusieurs formes selon les cultures et les traditions des peuples (Allin, 1990 ; Runte, 1997). Elles entretiennent les processus écologiques vitaux. Malheureusement les aires protégées sont sujettes de plus en plus à des pressions anthropiques. Dans plusieurs régions, ces pressions sur les écosystèmes en général et les aires protégées en particulier continuent de mettre en mal la biodiversité, accentuant la raréfaction voire la disparition de plusieurs ressources naturelles. Ces pressions sont d'autant plus acerbes qu'elles sont suscitées par des crises socio-politiques débouchant sur des conflits armés. En Afrique, les problèmes d'instabilité politique et les conflits entraînent une destruction de ces zones qui sont envahies par des populations fuyant les conflits (Mengue-Medou, 2002). Durant la guerre au Rwanda, le parc national Virunga est devenu un refuge pour des milliers de réfugiés qui fuyaient la guerre (Mengue-Medou., 2002). 
A l'Est du Congo une forte pression est exercée sur le Parc National de Kahuzi-Biega (PNKB), ce à la suite des guerres à répétition avec la présence de réfugiés rwandais remontant à 1994 et celle de multiples groupes armés. En 1997, le PNKB fut placé sur la liste des Sites du Patrimoine Mondial en péril (UNESCO, 2006). En Côte d'Ivoire, du fait des crises successives qu'a connues le pays au cours de ces dix dernières années, le PNC, la plus grande aire protégée du pays avec 1149450 ha a été déclaré patrimoine en péril (UNESCO, 2003). En effet, le sabotage et la désorganisation du système de gestion et de surveillance du PNC depuis le déclenchement de la crise en 2002, y ont accentué le développement de plusieurs activités d’origine anthropique. Parmi ces activités, on peut citer l’orpaillage, le braconnage, la transhumance par les grands troupeaux de bétails en provenance des pays de l'hinterland (Boutrais, 2008). Cependant les études traitant de l'impact des conflits sur sa biodiversité sont presque inexistantes. La flore et la végétation du Sud-Ouest du PNC ont déjà fait l'objet de plusieurs travaux. Parmi les plus récents, l'on peut signaler ceux se rapportant à la typologie de la végétation par une approche de signature spectrale de Kouassi et al. (2014). Les principales caractéristiques de la végétation et le potentiel de régénération des espèces ont été étudiés par Koulibaly (2008). N’Guessan (2009) a évalué la flore et caractérisé les changements survenus sur la végétation depuis septembre 2002. La présente étude s’intéressera uniquement aux fragments forestiers dans le Sud-Ouest du parc. Les objectifs visés étaient de faire l'inventaire de la flore des fragments forestiers, d'en évaluer la diversité floristique et de caractériser leur structure.

\section{Matériel et méthodes Site d'étude}

Situé au Nord-Est, le PNC est la plus grande aire protégée de la Côte d'Ivoire avec une superficie totale de 1149450 hectares. Il est situé dans la région administrative du Bounkani, à $570 \mathrm{~km}$ d'Abidjan entre les latitudes $8^{\circ} 30^{\prime}$ et $9^{\circ} 37^{\prime}$ 'Nord et les longitudes $3^{\circ} 07^{\prime}$ et $4^{\circ} 26^{\prime}$ Ouest (Figure 1). Son climat est de type tropical subhumide avec un régime de pluviométrie à une saison pluvieuse et une saison sèche (Novembre à Février). La pluviométrie totale annuelle était de 995,36 mm en 2015 (Yéo, 2016). Le relief est dominé par les plateaux d'une altitude moyenne de 300 mètres. Selon le substratum géologique et la topographie, les sols du PNC appartiennent aux sols de type ferralitiques, ferrugineux et hydromorphes. La végétation est dominée par les formations savanicoles avec des reliques forestières. Les formations savanicoles ont classiquement une physionomie qui varie avec la densité du couvert arboré et la taille des espèces ligneuses associées au tapis graminéen, le passage de l'une à l'autre étant souvent 
difficile à apprécier. Les reliques forestières sont des boisements denses représentés dans le parc par des îlots forestiers, qui occupent en général une position de plateau, les galeries forestières qui bordent les cours d'eau plus ou moins permanents et les forêts ripicoles qui occupent les rives du fleuve Comoé (Tahoux et al. 2006). Sa faune est caractérisée par une grande diversité d'espèces typiques aux savanes et d'espèces ubiquistes. Il renferme plus de trois quarts des espèces de grands mammifères de la Côte d'Ivoire. La densité de la population, dans la région Sud du parc, est passée à environ 10 habitants au km² dans les années 1980 (Wiese, 1988). En 1998, elle atteignait 13 habitants au $\mathrm{km}^{2}$ (INS, 2000). La principale activité de ces populations est l'agriculture.

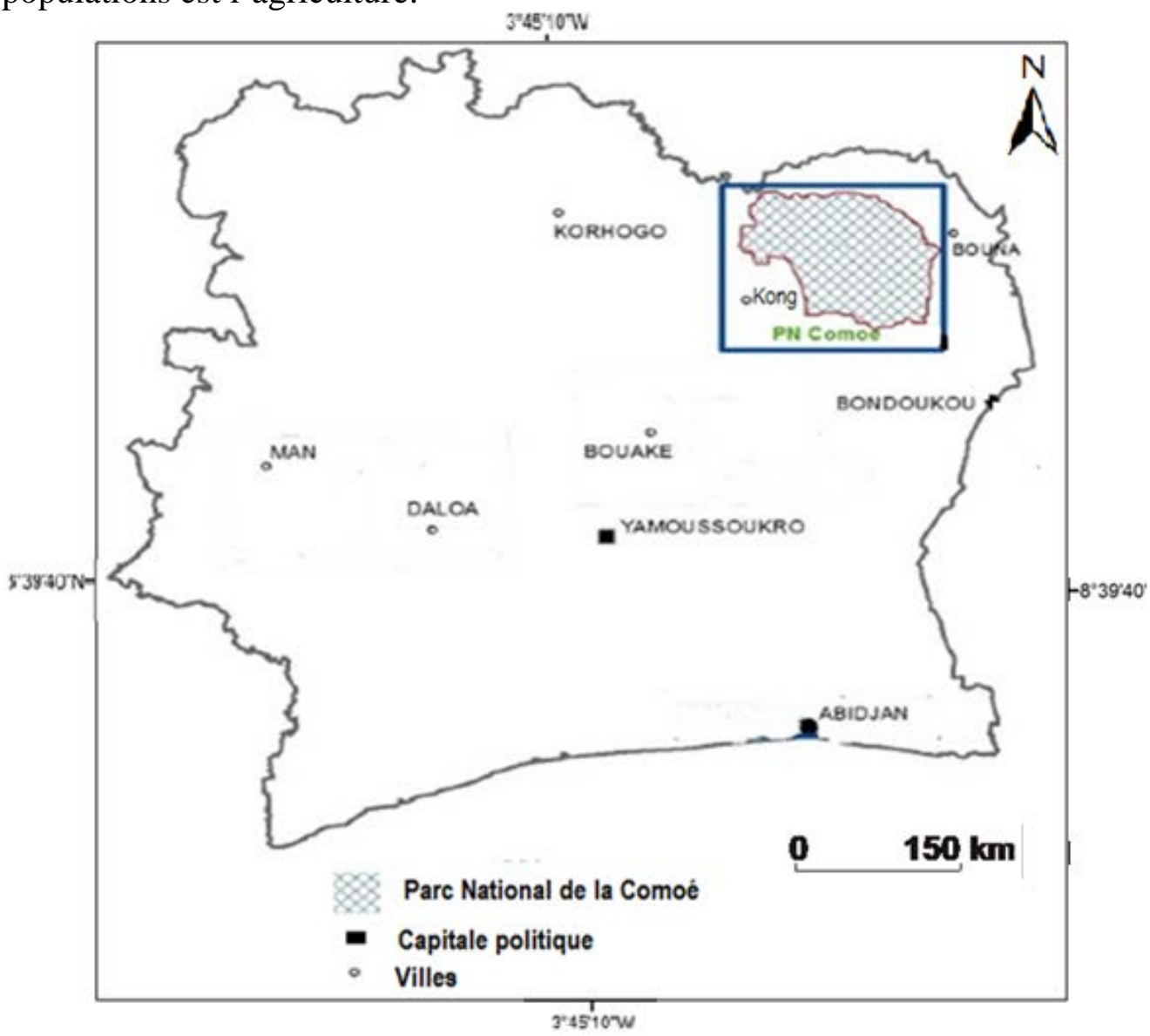

Figure 1 : Localisation de la zone d'étude dans le Sud-Ouest du Parc National de la Comoé. Location of the study area in the South West National Park Comoé.

\section{Collecte des données}

Sur la base d'une composition colorée $\operatorname{ETM}^{+}$(453) d'une image satellitaire Landsat $7 \mathrm{ETM}^{+}$de 2015, douze fragments forestiers ont été sélectionnés (Figure 2). Les coordonnées géographiques du point central de 
chacun des fragments ont été ensuite enregistrées dans un GPS (Global Positionning System) pour faciliter leur accès lors de la campagne de terrain.

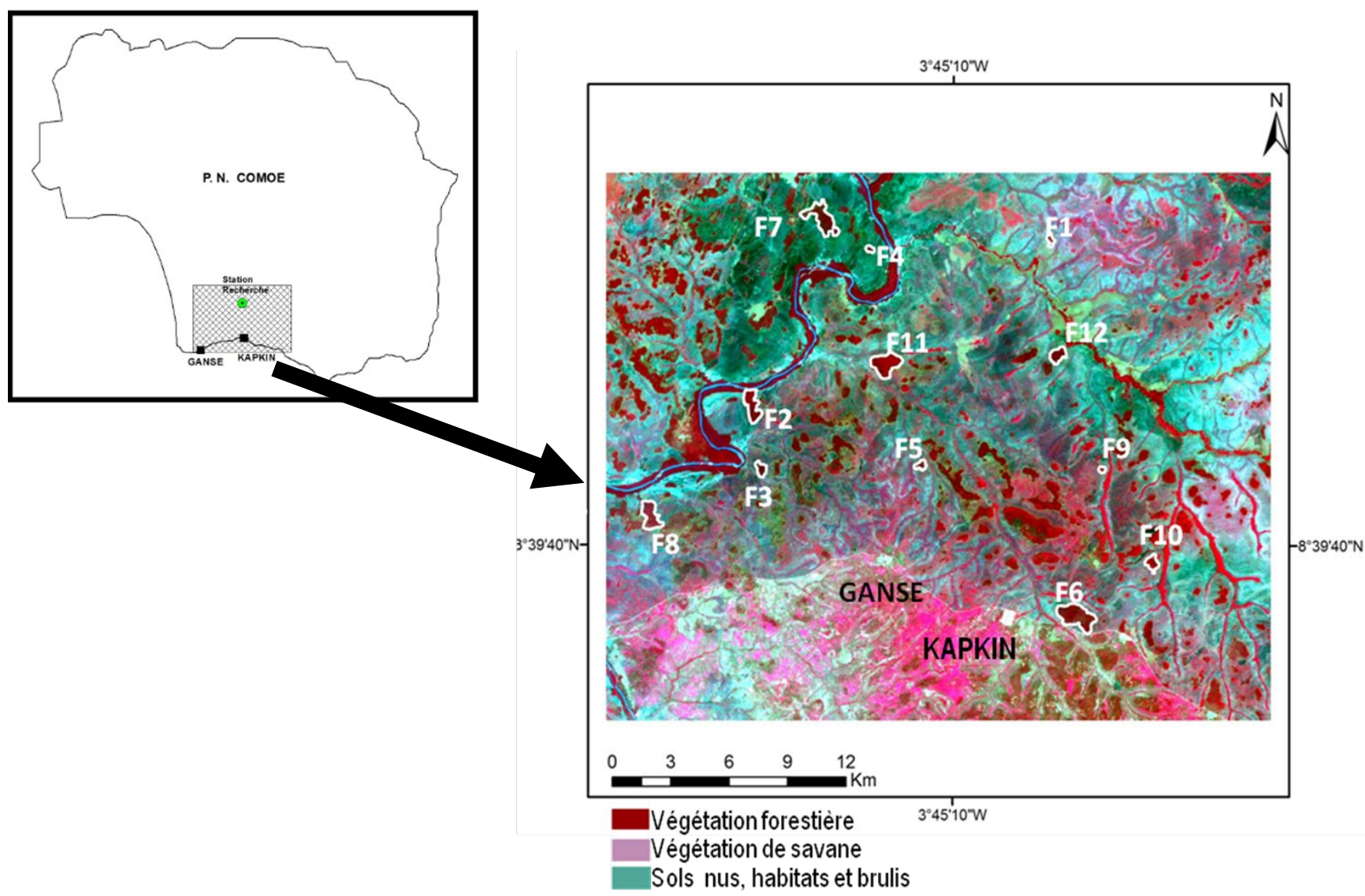

Figure 2 : Composition colorée $\mathrm{ETM}^{+}$(453) de l'image Landsat $\mathrm{ETM}^{+}$de 2014 montrant la répartition spatiale des fragments forestiers échantillonnés. Composite image using in order ETM+4, ETM+5 and ETM+3 bands from Landsat ETM image acquired in 2014, showing the spatial distribution of forest fragments sampled.

( $F=$ forêt. 1 à 12 : numéro de la forêt). Les contours des forêts sont indiqués en blanc.

La campagne de terrain s’est déroulée du 28 Juillet au 04 Août 2015 (soit 10 jours). La collecte des données proprement dite s'est faite à travers une série d'échantillonnages suivant des transects linéaires de $500 \mathrm{~m}$ de longueur auxquels s'ajoute une bande de $5 \mathrm{~m}$ de largeur de part et d'autre de la ligne du transect soit $10 \mathrm{~m}$ de largeur (Figure 3). La superficie totale de chaque transect échantillonné est donc $10 \mathrm{~m}$ x $500 \mathrm{~m}$ soit $5000 \mathrm{~m}^{2}$. Deux types de relevés ont été effectués sur chaque transect; d'une part les mesures des diamètres à hauteur de poitrine (dbh) et de hauteur de tous les ligneux dont la circonférence est supérieure ou égale à $30 \mathrm{~cm}$; et d'autre part, 
l'inventaire de toutes les espèces floristiques vasculaires présentes sur la surface échantillonnée.

La plupart des taxons ont été identifiés sur le terrain. Ceux dont l'identification était douteuse l'ont été au laboratoire grâce à l'Herbier du Centre national de floristique (CNF) de l’Université Félix Houphouët Boigny (Abidjan).

La nomenclature qui a servi à nommer les taxons est celle de Lebrun et Stork (1991-1997) ; Aké-Assi (2001 et 2002) et l'Union Internationale pour la Conservation de la Nature, (2015) actualisée.

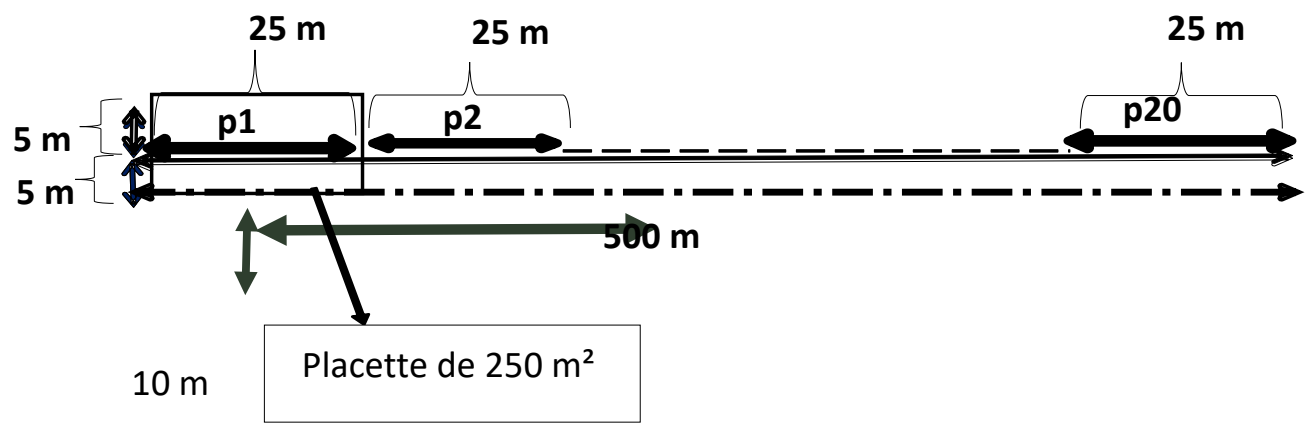

$25 \mathrm{~m}$

Figure 3 : Schéma d'un transect tel que réalisé sur le terrain pour la collecte des données. Diagram of a transect as carried out in the field for data collection

\section{Analyse des données}

Sur la base des listes floristiques obtenues, et de la liste UICN (2015), nous avons pu mettre en évidence la richesse floristique des fragments forestiers du PNC, ainsi que les espèces à statuts particuliers (espèces endémiques, rares, menacées d'extinction, espèces de grande valeur commerciale, etc.) qu'ils comportent.

Pour vérifier la ressemblance ou la dissemblance entre les différents fragments de forêt, nous nous sommes servis du coefficient de similitude de Sørensen (1948) dont l'expression mathématique est :

$$
\text { Cs }(\mathrm{s})=100 *(2 \mathrm{c} /(\mathrm{a}+\mathrm{b}))
$$

Où $\mathrm{a}=$ nombre d'espèces du milieu $\mathrm{A}, \mathrm{b}=$ nombre d'espèces du milieu $\mathrm{B}$ et $\mathrm{c}=$ nombre d'espèces communes aux deux milieux écologiques.

Les valeurs de Cs varient entre 0 et $100 \%$. Plus les listes ont des espèces en commun, plus Cs tend vers 100\%. Plus les deux listes floristiques sont dissemblables, plus la valeur de Cs tend vers 0 .

La diversité floristique quantitative du Sud-Ouest du PNC a été évaluée grâce à l'indice de diversité de Shannon (H) qui est communément utilisé à cet effet (Huston, 1995). Cet indice prend en compte la régularité d'abondance des espèces (Peet, 1974). Son expression mathématique est 
$\mathrm{H}=-\mathrm{Pi} \ln \mathrm{Pi}$ (Magurran, 1988)

Où Pi est la proportion des individus composant une espèce $i$.

La proportion est considérée ici comme le rapport de l'effectif des individus de l'espèce i sur l'effectif total des espèces. $\mathrm{H}$ varie entre Pi et ln de Pi.

La structure horizontale des peuplements a été analysée grâce au calcul de la densité de ligneux (D) et de celui de la surface terrière (g). La densité de ligneux (D), qui s'exprime par la formule suivante : $\mathrm{D}=\mathrm{N} / \mathrm{S}$

Avec $\mathrm{N}=$ nombre d'arbres du transect et $\mathrm{S}$ = superficie de la zone d'étude. D s'exprime en arbres/ha.

L'aire basale de tiges (A), qui se calcule selon l'expression suivante : $g=c^{2} / 4 \pi$

Pour un peuplement, la surface terrière $(G)$ est la surface que représenteraient tous les troncs des arbres d'un hectare de la forêt que l'on aurait coupé à 1,30 mètre de hauteur.

$\mathrm{G}=\sum_{1}^{i} g_{i}$

\section{Résultats et discussion}

Résultats

\section{Richesse floristique des fragments forestiers}

L’inventaire de la flore a permis de recenser 256 espèces végétales dans les fragments forestiers du Sud-Ouest. Ces espèces se répartissent en 179 genres et 62 familles. Les familles les plus importantes en termes de nombres d'espèces (Tableau 1) sont représentées par les Rubiaceae et les Fabaceae avec respectivement 20 et 17 des espèces recensées.

Tableau 1 : Liste des familles les plus abondantes des fragments forestiers échantillonnés dans le Sud-Ouest du Parc National de la Comoé. List of the most abundant families of forest fragments sampled in the South West of the Comoé National Park.

\begin{tabular}{ccc}
\hline Nom de famille & Nombre d'espèces & Pourcentage (\%) \\
\hline Rubiaceae & 20 & 8 \\
Fabaceae & 17 & 7 \\
Apocynaceae & 15 & 6 \\
Euphorbiaceae & 13 & 5 \\
Caesalpiniaceae & 11 & 4 \\
Mimosaceae & 9 & 3 \\
Autres familles & 171 & 67 \\
\hline
\end{tabular}

La répartition par affinité chorologique des espèces met en évidence une dominance des espèces guinéo-congolaises (GC, GCW et GCi) avec $48 \%$ suivi de celle des espèces de transition entre la zone guinéo-congolaise et la zone soudanienne (GC-SZ), avec 37\% (Figure 4). 


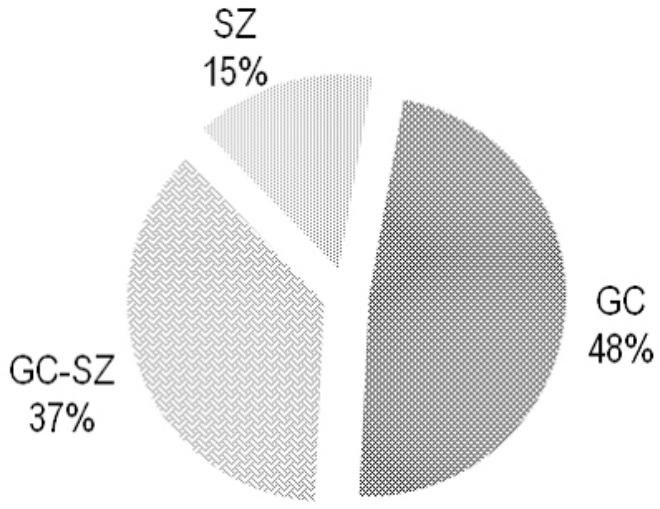

Figure 4 : Spectre de la répartition phytogéographique des espèces de la flore des fragments forestiers du Sud-Ouest du Parc National de la Comoé. Spectrum of the phytogeographical distribution of species from South West forest fragments of the National Park of Comoé.

GC-SZ = Taxon de la zone de transition entre la région guinéocongolaise et la région soudanienne ; $\mathrm{GC}=$ Taxon de la région guinéocongolaise ; GCW = Taxon endémique du bloc forestier à l'Ouest du Togo, comprenant le Ghana, la Côte d'Ivoire, le Libéria, la Sierra Leone, la Guinée, la Guinée Bissau, la Gambie et la Sénégal ; GCi = Taxon endémique à la Côte d'Ivoire; $\mathrm{SZ}=$ taxon de la région soudano-zambézienne

Parmi les espèces inventoriées, 14 sont endémiques au bloc forestier Ouest africain (GCW) dont 2 espèces endémiques à la flore ivoirienne (Tableau 2). Les endémiques ivoiriennes (GCI) sont : Salacia miegei N. Halle et Uvaria tortilis A. Chev.. Six (6) autres espèces inscrites sur la liste rouge de l’UICN (2015) sont vulnérables (Tableau 2). Parmi ces dernières, Salacia miegei N. Halle a été signalée uniquement dans le fragment forestier (F8). Quant à Uvaria tortilis A. Chev, elle se retrouve dans 9 fragments forestiers (F11 ; F9 ; F10 ; F5 ; F8 ; F7 ; F4 ; F3 ; F2) sur les 12 fragments inventoriés.

Tableau 2 : Liste des espèces endémiques dans le Parc National de la Comoé. List of endemic species reported in the National Park of Comoé.

\begin{tabular}{cccc}
\hline $\mathrm{N}^{\circ}$ & Espèces & Niveau d'endémisme & Statut UICN \\
\hline 1 & Alafia whytei & - & VU \\
2 & Afzelia bella & GCW & \\
3 & Albizia ferruginea & - & VU \\
4 & Amorphophallus accrensis & GCW & \\
5 & Cassipourea afzelii & GCW & \\
6 & Cola caricaefolia & GCW & LC \\
7 & Commelina erecta & - & LR \\
8 & Cordia millenii & - & \\
9 & Dalbergia oblongifolia & GCW & \\
\hline
\end{tabular}




\begin{tabular}{lccc}
\hline 10 & Dicliptera elliotii & GC-SZ & LC \\
11 & Drypetes afzelii & GCW & VU \\
12 & Elytrophorus spicatus & - & LC \\
13 & Eugenia leonensis & GCW & VU \\
14 & Khaya senegalensis & - & \\
15 & Kolobopetalum leonense & GCW & LR \\
16 & Milicia excelsa & - & \\
17 & Sabicea discolar & GCW & VU \\
18 & Salacia miegei & GCi & \\
19 & Sapium carterianum & GCW & \\
20 & Tricalysia discolor & GCi & \\
21 & Uvaria tortilis & GCW & VU \\
22 & Vangueriella vanguerioides & - & Vitellaria paradoxa
\end{tabular}

GCi : endémique à la flore ivoirienne ; GCW : Bloc forestier ouest africain ; LR : Risque faible; VU = vulnérables ; UICN = Union Internationale pour la Conservation de la Nature;

LC: préoccupation mineure.

Par ailleurs, la flore des fragments forestiers du Sud-Ouest du PNC révèle la présence d'espèces de grande valeur commerciale, soit 9 essences (Tableau 3). Ces essences forestières ont été subdivisées en trois catégories sur la base du critère de commercialisation en Côte d'Ivoire SODEFOR, (1993). Selon SODEFOR (1993), 4 de ces essences constituent des essences principales qui sont couramment commercialisées (catégorie 1), 3 sont sporadiquement commercialisées (catégorie 2) et 2 sont à promouvoir (catégorie 3).

Tableau 3 : Liste des essences relevées dans le PNC et identifiées comme exploitables en tant que bois d'œuvres en Côte d'Ivoire par SODEFOR, (1993). List of species identified as softwood lumber in Côte d'Ivoire by SODEFOR (1993) reported in the PNC.

\begin{tabular}{ccr}
\hline Espèces & Familles & Catégorie \\
\hline Afzelia bella var. gracilior & Caesalpiniaceae & 1 \\
Albizia ferruginea & Mimosaceae & 2 \\
Antiaris toxicaria var. welwitschii & Moraceae & 1 \\
Ceiba pentandra & Bombacaceae & 1 \\
Celtis milbraedii Engl. & Ulmaceae & 2 \\
Celtis zenkeri EngI. & Ulmaceae & 3 \\
Detarium senegalense & Caesalpiniaceae & 3 \\
Milicia excelsa & Moraceae & 1 \\
Ricinodendron heudelotii & Euphorbiaceae & 2 \\
\hline
\end{tabular}

\section{Diversité floristique}

Les valeurs des indices de Shannon et Weaver (1948) des fragments forestiers varient de 3,5 à 4,5 (Tableau 4). Elles indiquent que les forêts F9, F10, F8 et F6 sont les plus diversifiées avec des valeurs respectives de l'ordre de 4,5 ; 4,24 ; 4,12 et 4,07. La forêt F2 est la moins diversifiée avec une valeur de 3,5. 
Tableau 4 : Valeur de l'indice de diversité de Shannon et Weaver des différents fragments forestiers échantillonnés dans le Sud-Ouest du Parc National de la Comoé. Values of Shannon and Weaver index corresponding to the different forest fragments sampled in the South West of the National Park of Comoé.

\begin{tabular}{lllllllllllll} 
Fragments & F1 & F2 & F3 & F4 & F5 & F6 & F7 & F8 & F9 & F10 & F11 & F12 \\
Shannon & 3,7 & 3,5 & 3,7 & 3,6 & 3,6 & 4 & 3,7 & 4,1 & 4,5 & 4,2 & 3,6 & 3,7 \\
\hline
\end{tabular}

Le tableau 5, présente le degré de ressemblance entre les différents fragments de forêts. Les coefficients de similitude calculés entre les différents milieux indiquent des taux variant entre 32 et $64 \%$. Le plus grand taux de ressemblance (64\%) est obtenu entre les forêts F3 et F4 tandis que la plus forte dissemblance (32\%) a été obtenue entre F10 et F1. Cependant, la plupart des fragments forestiers se ressemblent floristiquement (Tableau 5).

Tableau 5 : Matrice des coefficients de similitude entre les différents fragments forestiers du Sud-Ouest du Parc National de la Comoé. Matrix of similarity coefficient between the different forest fragments in the South West of the National Park of Comoé.

\begin{tabular}{cccccccccccc}
\hline & F1 & F2 & F3 & F4 & F5 & F6 & F7 & F8 & F9 & F10 & F11 \\
\hline F2 & 51 & & & & & & & & & & \\
F3 & 51 & 61 & & & & & & & & & \\
F4 & 52 & 61 & 54 & & & & & & & & \\
F5 & 49 & 58 & 48 & 42 & & & & & & & \\
F6 & 44 & 52 & 47 & 44 & 52 & & & & & & \\
F7 & 41 & 54 & 47 & 55 & 44 & 53 & & & & \\
F8 & 51 & 51 & 57 & 56 & 46 & 50 & 48 & & & \\
F9 & 38 & 41 & 39 & 39 & 38 & 45 & 35 & 59 & & & \\
F10 & 32 & 43 & 37 & 39 & 41 & $\mathbf{6 4}$ & 37 & 48 & 50 & & \\
F11 & 51 & 60 & 53 & 42 & 48 & 37 & 39 & 41 & 36 & 35 & \\
F12 & 54 & 57 & 58 & 56 & 48 & 51 & 52 & 53 & 42 & 39 & 57 \\
\hline \multicolumn{8}{c}{ F=forêt ; F1 à F12 = forêt 1 à forêt 12 } & & &
\end{tabular}

\section{Structure des fragments forestiers}

La figure 5 A représente la densité en arbres des douze fragments forestiers échantillonnés. Elle montre que les forêts F1, F2 et F3, qui comportent respectivement 724, 676 et 616 tiges/ha sont les plus denses. Et la forêt F12 avec 306 tiges/ha est la moins dense. En ce qui concerne les aires basales (Figure $5 \mathrm{~B}$ ), ce sont les forêts F12 (30 m²/ha) et F3 $\left(26 \mathrm{~m}^{2} / \mathrm{ha}\right)$ qui ont les aires basales les plus élevées. Les forêts F11 et F13 qui ont respectivement $12 \mathrm{~m}^{2} /$ ha et $13 \mathrm{~m}^{2} / \mathrm{ha}$, ont les valeurs d'aire basale les plus faibles. La figure 6 indique que la densité de tiges n'est pas liée à l'aire basale dans les formations inventoriées $\left(\mathrm{R}^{2}=0,1678\right)$. 


\section{Densité (tiges/ ha)}

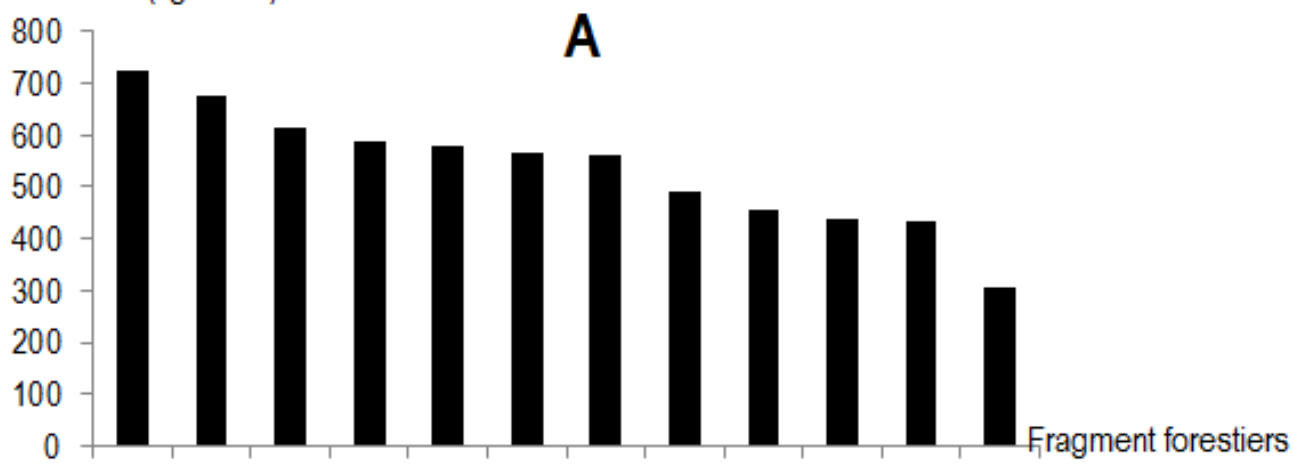

$\begin{array}{llllllllllll}\text { F1 } & \text { F2 } & \text { F3 } & \text { F4 } & \text { F5 } & \text { F6 } & \text { F7 } & \text { F8 } & \text { F9 } & \text { F10 } & \text { F11 } & \text { F12 }\end{array}$

\section{Aire basale $\mathrm{m}^{2} / \mathrm{ha}$}

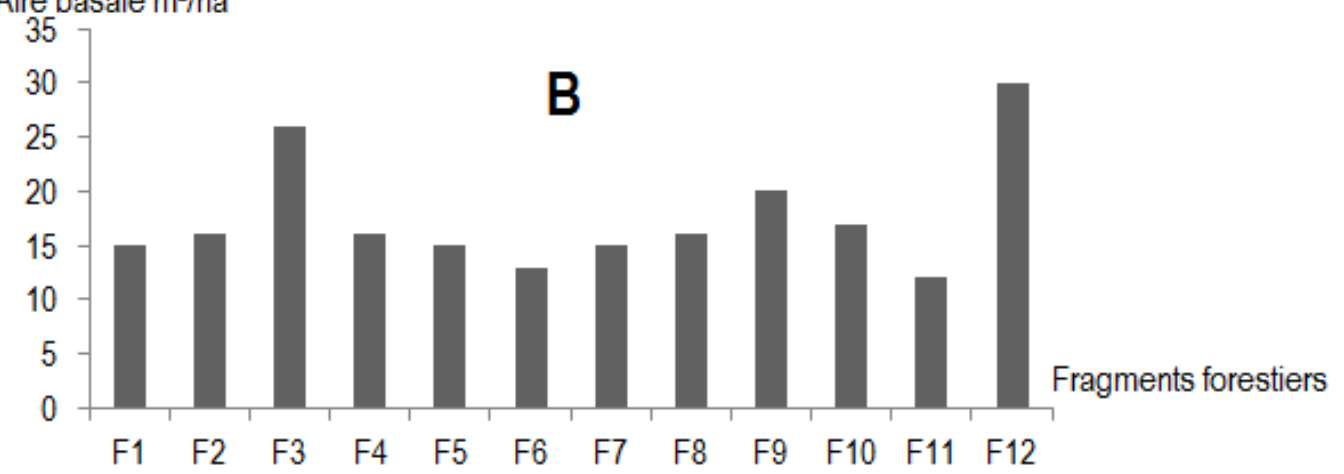

Figure 5: Densités de tiges (A) et aires basales moyennes (B) des différentes formations forestières échantillonnées dans le Parc National de la Comoé. Stem densities (A) and the mean basal area (B) of the different forest types sampled in the National Park of Comoé.

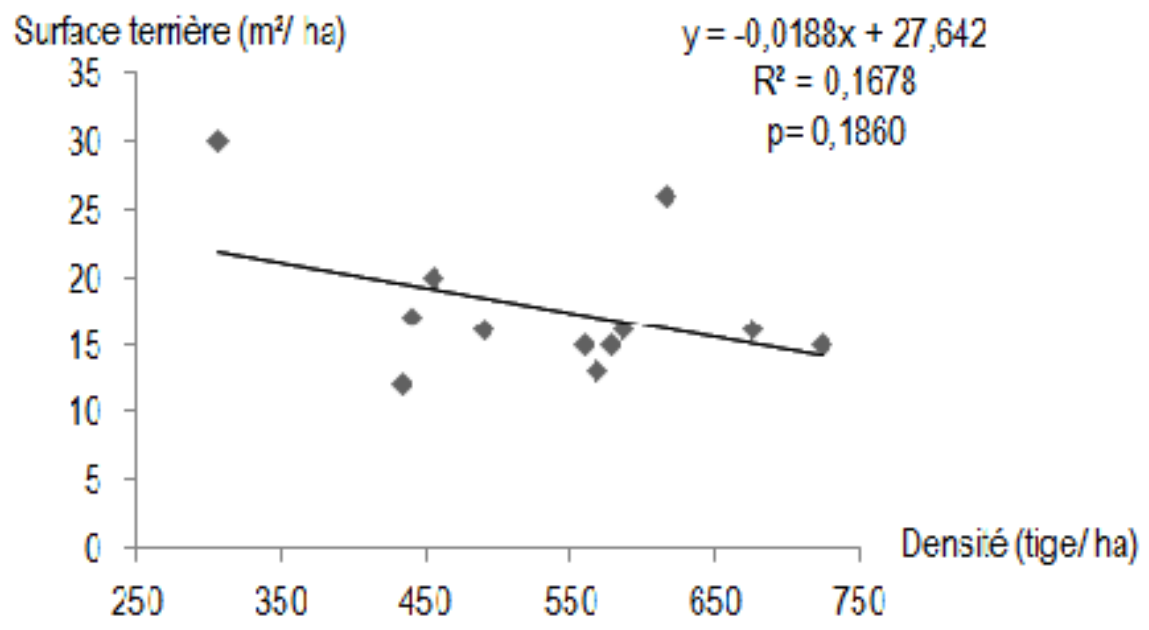

Figure 6 : Régression linéaire entre la densité des tiges et l'aire basale des différentes formations forestières échantillonnées. Linear regression between stem density and basal area of different forest fragments sampled 
L'histogramme comparatif du nombre d'arbres par strate (Figure 7), indique que les arbres sont inégalement distribués dans les différentes classes de hauteurs dans les formations échantillonnées. La proportion de la strate inférieure $(\mathrm{h}<8 \mathrm{~m}$ ) est la plus élevée dans toutes les formations forestières (Figure 7). Cette strate est suivie de la strate moyenne $(8<\mathrm{h}<32 \mathrm{~m})$. La strate supérieure $(\mathrm{h}>32 \mathrm{~m}$ ) est la moins représentée au sein des forêts. Les espèces qui composent cette dernière strate sont en général, Schrebera arborea, Albizia zygia, Cola cordifolia, Cola caricaefolia, Ceiba pentandra, Milicia excelsa, Afraegle paniculata, Celtis zenkeri, Balanites wilsonian, Manilkara multinervis, Anogeissus leiocarpus, Celtis integrifolia, Cordia senegalensis, Cynometra megalophylla, Ficus ovata, Allanblackia floribunda, Alafia multiflora, Antiaris toxicaria var. africana, Morus mesozygia, Tamarindus indica, Mimusops kummel, Albizia ferruginea, Combretum nigricans, Erythrophleum guineense, Parkia biglobosa, Lannea nigritana, Salacia staudtii Cassipourea barteri, etc.

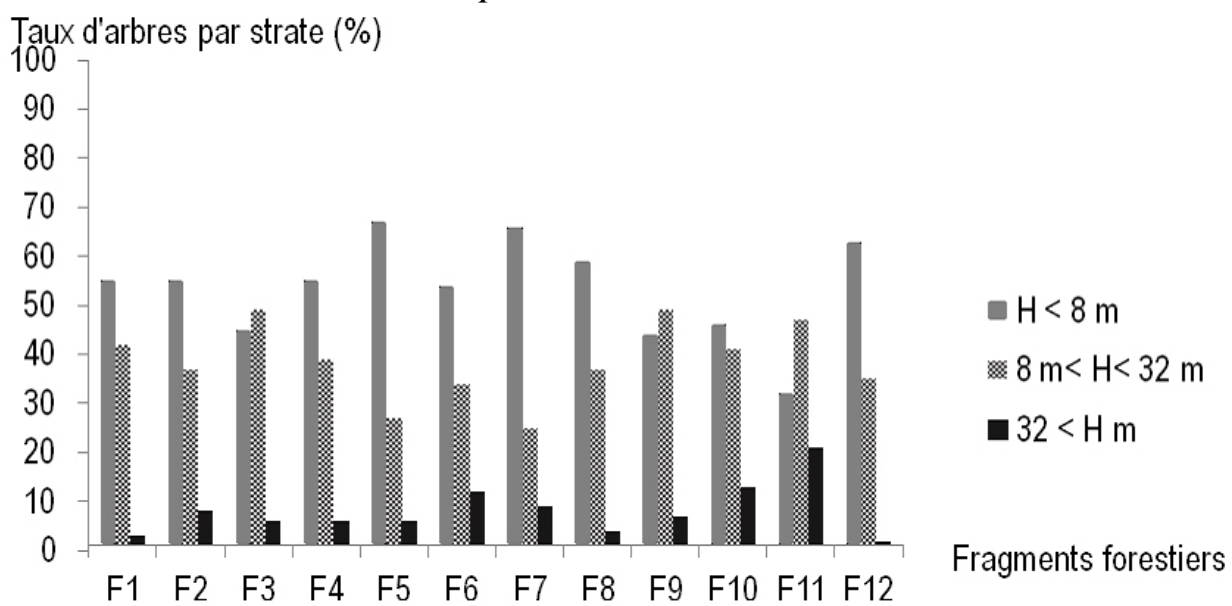

Figure 7: Histogramme comparatif des strates au sein des fragments forestiers inventoriés dans le Sud-Ouest du Parc National de la Comoé. Layers of comparative histogram in forest fragments surveyed in the South West National Park Comoé.

\section{Discussion}

\section{Richesse et diversité floristique}

Les investigations effectuées dans les fragments de forêts du SudOuest du Parc National de la Comoé au cours de cette étude, ont permis de recenser 256 espèces reparties entre 179 genres et 62 familles. Par contre, les travaux de N'Guessan (2009), sur l'ensemble du PNC ont estimé la flore totale à 1001 espèces reparties en 562 genres et 131 familles. Cet écart au niveau du nombre d'espèces, genres et familles peut s'expliquer par le fait que la superficie couverte par la présente étude est estimée à environ 175 
000 ha soit 1/7 de la superficie totale du PNC et que ces travaux n'ont concerné que les fragments de forêts.

Les Rubiaceae sont la famille la plus dominante suivie des Fabaceae, des Apocynaceae, des Euphorbiaceae et des Poaceae. Par contre, les travaux de N’Guessan (2009), ont révélé que les Poaceae constituent la famille la plus abondante de la flore du PNC. La prépondérance des Rubiaceae dans ces travaux, serait due au fait que les relevés n’ont concerné que les fragments forestiers. Ces résultats corroborent ceux d’Aké-Assi (2002), selon lesquels les Rubiaceae seraient abondantes dans les zones de forêts sèches ou humides de Côte d'Ivoire. Nous en déduisons donc que les fragments forestiers à l'intérieur du PNC sont floristiquement proches de certaines forêts denses du Sud du pays. Par ailleurs, la prédominance des Poaceae indiquée par N'Guessan (2009), serait liée au fait que les savanes constituent la matrice essentiel du PNC. Car selon Makanga (2011), les savanes seraient habituellement dominées par les Poaceae. En effet, N’Guessan (2009) dans ses travaux n’aurait pas privilégié un type de végétation par rapport à un autre. Bien que la présente étude n'a concerné que le 1/7 de la surface totale du PNC, 23 espèces à statuts particulier ont été recensées. Parmi elles, 14 espèces sont endémiques à l'Afrique de l'Ouest dont 02 endémiques à la Côte d’Ivoire (Salacia miegei N. Halle et Uvaria tortilis A. Chev. Ex Hutch. \& Dalziel) et les 11 autres figurent sur la liste rouge de l'UICN (2015). N'Guessan (2009) avait signalé 46 espèces à statuts particuliers dont 10 endémiques à la flore Ouest Africaine et une (01) seule espèce endémique ivoirienne (Cissus touraensis A. Chev.). Neuf (9) autres espèces sont des essences forestières de grande valeur commerciale en Côte d’Ivoire pour la qualité de leur bois.

Certes, la liste de la floristique obtenue peut encore être améliorée par d'autres études. Toutefois, les résultats obtenus ont permis d'améliorer les connaissances sur la flore du PNC surtout la flore endémique. En effet, parmi les 14 espèces GCW inventoriées dans cette étude, aucune ne figure sur liste établie par N’Guessan (2009). Ainsi le nombre d'espèces endémiques à la flore ivoirienne recensées dans cette aire protégée est passé à 3 à ce jour. Hormis les milieux échantillonnés (milieux forêts), ces résultats pourraient trouver leur justification dans les méthodologies utilisées. En effet, notre méthodologie a associé la technique d'échantillonnage par le transect linéaire à celle du quadrat. Les forêts regorgeraient davantage d'espèces à statut particulier. Ces résultats laissent supposer que le PNC n'a pas subi de grands dommages d'origine anthropique, car, selon Tchouto (2004) et Van Gemerden (2004), les espèces à statuts particuliers seraient les plus sensibles aux perturbations causées par l'homme. Leur présence serait de ce fait, signe d'absence d'activités humaines permanentes telles que l'agriculture sur brûlis, l’orpaillage ou 
encore une sédentarisation au sein des fragments forestiers, car ces activités sont les plus perturbatrices de la flore. Parmi ces dernières, l'agriculture demeure la plus spectaculaire, par la réduction intense de la couverture végétale qu'elle peut entraîner et les conséquences sur la reconstitution des forêts (Koulibaly, 2008). Quant au feu, bien qu'étant quelque fois d’origine humaine, il intervient pendant la saison sèche, permettant ainsi de maintenir la savane et assurer une bonne régénération de la flore herbacée en particulier pour la consommation des grands troupeaux d'herbivores du PNC. Les valeurs des indices de Shannon $(4,5 ; 4,24 ; 4,12$ et 4,07 respectivement pour les fragments F9, F10, F8 et F6) indiqueraient que les forêts du PNC en général sont assez diversifiées, ce qui confirme les travaux de Kouadio et al. (2006). Ces fragments sont caractérisés par la dominance de certaines espèces. Ainsi, ces forêts, sont le plus souvent dominées par une ou deux espèces. C'est le cas par exemple de Cynometra megalophylla Harms dans le fragment F12, Dialium guineense Willd et Detarium senegalense J.F. Gmel, dans le fragment F9, Diospyros abyssinica (Hiern) white et Anogeissus leiocarpus (DC.) Guill. \& Perr dans le fragment F1. Ce résultat s’expliquerait entre autre par le passage du feu dans le PNC. Ce feu aurait ouvert et morcelé l'ancien couvert forestier, ce qui a contribué à rendre plus riche la flore du parc (N'Guessan, 2009). De ce fait, outre les taxons de la région guinéo-congolaise (GC) (42\%), on note une forte présence d'espèces de transition entre la zone guinéo-congolaise et la zone soudanaise (GC-SZ) (37\%). Cette présence des espèces (GC-SZ) indiquerait que les fragments forestiers du parc, n’ont pas la même phénologie que leurs homologues du sud du pays (domaine guinéen) ; ce qui fait des fragments forestiers du PNC, des forêts denses relativement plus sèches et donc caractérisées par la perte quasi-totale des feuilles des arbres pendant la saison sèche, d'où une exacerbation des feux en cette période. Ces résultats confirment les travaux de Maley (1987) qui indiquent que le PNC ne comporte actuellement plus de forêts denses humides. L'analyse des coefficients de similitude entre les différents fragments forestiers, indique que la majorité des fragments forestiers sont semblables du point de vue floristique. Cette ressemblance serait liée au fait que ces fragments sont sous la dépendance d'un même climat et aussi des mêmes conditions édaphiques. Les formations forestières du PNC, seraient issues de grands blocs forestiers qui existaient dans le parc. En effet, compte tenu des feux de brousses récurrents, ces formations se seraient dissociées faisant place à la savane. Ainsi, la présence des espèces comme Anogeissus leiocarpus (DC.) Guill. \& Perr, Tamarindus indica Linn, Terminalia macroptera Guill. \& Perr, Diospyros mespiIiformis Hochst. ex A. DC. etc, aussi bien dans les fragments forestiers que dans les savanes pourrait confirmer ce résultat. 


\section{Structure verticale et horizontale des formations}

La densité moyenne en tiges des forêts (536 arbres/ha), s'expliquerait par le fait que les arbres rencontrés dans les fragments de forêts ont de petits diamètres. De façon générale, le nombre d'arbres varierait avec leur grosseur dans les milieux. C'est ce qui explique la faible densité d'arbre de la forêt F12 (306 arbres/ha). Cette forêt est dominée essentiellement par une espèce végétale ripicole (Cynometra megalophylla Harms) avec des gros diamètres et un sous-bois pauvre en jeunes tiges. Cette espèce est aussi indicatrice des forêts galeries selon N'Guessan (2009). La forêt F12 est donc une forêt galerie et les 11 autres, des ilots forestiers avec des densités allant de 434 à 724 arbres/ha. Ces résultats semblent être en désaccord avec ceux de Kouassi et al. (2014) qui stipulent que les îlots forestiers sont plus denses que les forêts galeries. Les résultats de cette étude indiquent que les forêts du PNC seraient plus denses (536 arbres/ha) que celles rencontrées dans le Parc National de la Marahoué (PNM), et la Forêt Classée du Haut Sassandra (FCHS). En effet, ces deux aires protégées, infiltrées par les populations pour des activités agricoles, ont des densités respectives de 334 arbres/ha (N'da et al. 2008) et 380 arbres/ha (Kouakou et al. 2015). Ce constat laisse deviner que lorsque la forêt n'est pas sous l'influence des activités humaines, elle peut conserver un grand nombre de tiges et un nombre important d'arbres (Aubréville, 1964 ; Kouakou et al., 2015). En effet, pour les pratiques agricoles ou l'orpaillage par exemple, les populations défrichent et abattent les arbres.

Au niveau des aires basales, les forêts F12 et F3 avec respectivement 30 et $26 \mathrm{~m}^{2} / \mathrm{ha}$ possèdent les valeurs des aires basales les plus élevées, contrairement aux forêts les plus denses qui ont de faibles aires basales. De ce fait, dans les différentes formations forestières, les tiges ont des circonférences relativement petites. La valeur plus élevée de l'aire basale de la forêt F12, s'expliquerait par la présence de plus de 80 pieds de Cynometra megalophylla, avec des circonférences comprises entre 150 et $300 \mathrm{~cm}$. A l'opposé, la forêt F1 bien que plus dense (724 tiges/ha) n'a qu'une surface terrière de $16 \mathrm{~m}^{2} / \mathrm{ha}$ à cause des petits diamètres des arbres qu'elle comporte ; ce qui justifie une faible corrélation entre la densité de tiges et l'aire basale.

La structuration des forêts en strate montre la dominance de microphanérophytes au sein de toutes les formations forestières inventoriées. Ces résultats influencent la densité des milieux. En effet la forte densité des arbres ne favorise pas une bonne aération dans les formations naturelles. Ces formations ne bénéficient d'aucun suivi humain. La compétition concurrence entre les individus pour la lumière et les éléments minéraux, ralentit leur croissance. Tandis que pour les arbres des plantations (plantations forestières), ils bénéficient de l'entretien et du suivi des sylviculteurs, 
notamment les éclaircies qui favorisent une meilleure aération et un bon développement des arbres.

\section{Conclusion}

Bien que situé en zone sous contrôle des forces rebelles aux Autorités gouvernementales, le Parc National de la Comoé continue d'abriter une flore diversifiée malgré les conflits de 2002 à 2011. Au total, une liste de 256 espèces végétales regroupées en 179 genres et 62 familles dont la famille des Rubiaceae est la plus prépondérante, ont été recensées lors de cette étude. Parmi ces espèces, 23 ont un statut particulier. L'analyse des fragments forestiers au niveau de la structure, a mis en évidence que les ilots forestiers sont les formations forestières les plus denses du parc avec plus de 550 arbres/ha. Cependant, leurs surfaces terrières sont relativement faibles et ne sont pas corrélées aux densités des tiges. Cette flore qui est dominée par les espèces guinéo-congolaises (GC) contient un nombre remarquable d'espèces de transition entre la région guinéo-congolaise et la région soudanienne (GC-SZ).

Il ressort que les conflits n’ont pas eu un impact assez significatif sur la flore forestière en général et en particulier sur les espèces endémiques qu'elle comporte. Cette aire protégée est d'une importance écologique capitale compte tenu du nombre important d'espèces végétales à statut particulier rencontrées. Les résultats obtenus dans cette étude devraient interpeller les autorités compétentes à prendre des décisions urgentes et efficaces pour une meilleure protection du parc national de Comoé.

\section{Remerciements}

La présente étude a été réalisée dans le cadre du projet D2PCPCI financé dans le cadre du C2D avec l'appui technique de l'Institut de Recherche pour le Développement (IRD). L'étude a été possible grâce à l'accord de l'Office Ivoirien des Parcs et Réserves (OIPR) qui a autorisé l'accès au Parc National de la Comoé et aux gestionnaires de la Station de Recherche en Ecologie.

\section{References :}

1. Aké-Assi L., 2001. Flore de la Côte d’Ivoire 1, catalogue, systématique, biogéographie et écologie. Genève, Suisse : Conservatoire et jardin Botanique de Genève ; Boissiera 57 : 396 p.

2. Aké-Assi L., 2002. Flore de la Côte d’Ivoire 2, catalogue, systématique, biogéographie et écologie. Genève, Suisse Conservatoire et Jardin Botanique de Genève, Boissiera 58 : 441 p

3. Allin CW., (1990). International Handbook of National Parks and Nature Reserves. Greenwood Press, Greenwood, CT, USA, 1-20 
4. Aubréville A., 1964. Végétation et flore comparées dans l'Inde et l'Afrique tropicale. Museum National d'Histoire Naturelle, 208-215.

5. Boutrais J., 2008. Pastoralisme et aires protégées d'Afrique de l'Ouest en regard de l'Afrique de l'Est. Aires protégées, espaces durables, 215-246.

6. Huston MA., 1995. Biological diversity: The coexistence of species on changing andscapes. Cambridges University press. $708 \mathrm{p}$

7. I.N.S 2000. Données socio-démographiques et économiques des localités. Institut National de la Statistique Tome I : Résultats définitifs par localité : Région du Zanzan. Recensement général de la population et de l'habitat 1988/2000, (3). Abidjan

8. Kouadio HB., Tahoux T. \& Biemi J., 2006. Apport de la télédétection et d'un système d'information géographique (SIG) à la gestion intégrée et participative des aires protégées en Côte d’Ivoire : application au parc national de Comoé (réserve de biosphère). Étude financée par le programme UNESCO/MAB pour Jeunes Scientifiques 2006, $60 \mathrm{p}$

9. Kouakou KA., Barima YSS., Kouakou ATM., Sangne YC., Bamba I. \& Kouamé NF., 2015. Diversité végétale post-conflits armés de la Forêt Classée du Haut-Sassandra (Centre-Ouest de la Côte d’Ivoire). Journal of Animal \& Plant Sciences, 26(2) : 4058-4071.

10. Kouassi KE., Sangne YC \& Dibi N’H., 2014. Typologie de La végétation par une approche de signature spectrale dans le Sud du Parc National de La Comoé (Nord-Est Côte d'Ivoire). European Scientific Journal.10 (36) : 1857 - 7881

11. Koulibaly A., 2008. Caractérisation de la végétation et dynamique de la régénération, sous l'influence de l'utilisation des terres, dans des mosaïques forêts-savanes, des régions de la réserve de Lamto et du Parc National de la Comoé, en Côte d’Ivoire. Thèse de doctorat unique, Université. Cocody ; 208 p.

12. Lebrun JP. \& Stork AL., 1997. Énumération des plantes à fleurs d'Afrique tropicale: Gamopétales : Ericaceae à Lamiaceae, 4, Conservatoire et Jard. Bot., Genève, 712 p.

13. Makanga JDM., 2011. Mosaïque forêt-savane et exploitation des ressources forestières du Gabon. Geo-Eco-Trop, 35 :41-50.

14. Maley J., 1987. Fragmentation de la forêt dense humide africaine et extension des biotopes montagnards au Quaternaire récent: nouvelles données polliniques et chronologiques. Implications paléoclimatiques et biogéographiques. Paleocology of Africa, 18 : 307-334.

15. Magurran AE., 1988. Ecological Diversity and its Measurement. Croom Helm, London, 192 p 
16. Mengue-Medou C., 2001. Les aires protégées en Afrique : perspectives pour leur conservation, VertigO - la revue électronique en sciences de l'environnement [En ligne], Volume 3 Numéro 1; consulté le 27 décembre 2016. URL : http://vertigo.revues.org/4126 ; DOI : 10.4000/vertigo.4126

17. N’da DH., Adou CY., N’guessan K.E., Koné M. \& Sangne YC., 2008. Analyse de la diversité floristique du parc national de la Marahoué, Centre-Ouest de Côte d'Ivoire. Afrique Science, 4 : 552579

18. N’Guessan KE., 2009. Projet d'élaboration d'une base de données numérique sur la flore et la végétation du parc national de la Comoé, au nord-est de la cote d'ivoire, $37 \mathrm{p}$.

19. Peet RK., 1974. The measurement of species diversity. Annuual Review of Ecology and Systematics 5 : 285 - 307.

20. Runte A., 1997. National parks: the American experience. University of Nebraska Press, Lincoln, Nebraska, USA.335p

21. Shannon CE. \& Weaver W., 1948. The mathematical theory of communication.univ.Illinois Press, Urbana, 117 p.

22. Sørensen T., 1948. A method of establishing groups of equal amplitude in plant sociology based on similitary of species content. Det Kongelige Danske Videnskabernes Selskab. Biologiske Skrifter,5,4: 1-34.

23. SODEFOR., 1993. Règles de culture et d'exploitation en forêt dense de Côte d'Ivoire. Rep.CI. Abidjan. 54p

24. UNESCO., 2003. Convention concernant la protection des patrimoines mondiaux culturels et naturels comité du patrimoine mondial : Vingt-septième session. Paris, Siège de l'UNESCO, Salle XII 30 Juin - 5 Juillet 2003-.173p

25. UNESCO., 2006. Décisions adoptées lors de $30^{\text {ème }}$ session du comité du patrimoine mondial (Vilnius, 2006); 201p. (http://whc.unesco.org/archive/2006/whc06-30com-19f.pdf)

26. Tchouto GPM. 2004. Plant diversity in Central African rain forest: implication for Biodiversity conservation in Cameroon. PhD.Thesis, Department of plant sciences, Biosystematic Group, Wageningen University, 208 p.

27. Tahoux TM., Angui P., Konaté S., Goh D., Agnissan A. \& Guehi I., 2006. Le Parc National de la Comoé. Réserve de biosphère et patrimoine mondial. Notes sur le milieu naturel et données socioéconomiques. $38 \mathrm{p}$.

28. Van Gemerden B.S., 2004. Disturbance, diversity and distributions in Central Africain rain forest. Ph-D. thesis, Wageningen University, 199 p. 
29. UICN., 2015. Red List of Threatened Species, International Union for Conservation of Nature Version 2015.

30. Wiese B., 1988. Elfenbeinküste: Erfolge und Probleme eines Entwicklungslandes in den westafrikanischen Tropen. In Wissenschaftliche. Länderkunden. Storkebaum,W. 29 : 1-303.

31. Yéo ZS., 2016. Diversité floristique et structure des fragments forestiers du Sud-Ouest du Parc National de la Comoé après les conflits des années 2000 en côte d'ivoire. Mémoire de master, Option: Ecologie et Gestion Durable des Ecosystèmes, UFR Environnement, Université Jean Lorougnon Guédé (Daloa, Côte d'Ivoire), $54 \mathrm{p}$. 